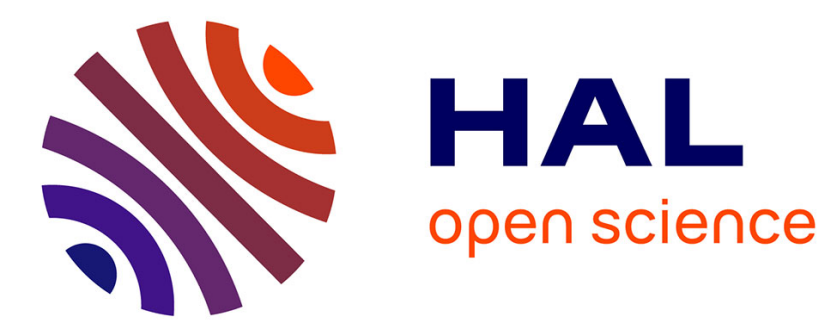

\title{
Direct measurement of the thermomechanical Lehmann coefficient in a compensated cholesteric liquid crystal
} Patrick Oswald, Alain Dequidt

\section{To cite this version:}

Patrick Oswald, Alain Dequidt. Direct measurement of the thermomechanical Lehmann coefficient in a compensated cholesteric liquid crystal. EPL - Europhysics Letters, 2008, 83 (1), pp.16005. 10.1209/0295-5075/83/16005 . ensl-00351719

HAL Id: ensl-00351719

https://hal-ens-lyon.archives-ouvertes.fr/ensl-00351719

Submitted on 10 Jan 2009

HAL is a multi-disciplinary open access archive for the deposit and dissemination of scientific research documents, whether they are published or not. The documents may come from teaching and research institutions in France or abroad, or from public or private research centers.
L'archive ouverte pluridisciplinaire HAL, est destinée au dépôt et à la diffusion de documents scientifiques de niveau recherche, publiés ou non, émanant des établissements d'enseignement et de recherche français ou étrangers, des laboratoires publics ou privés. 


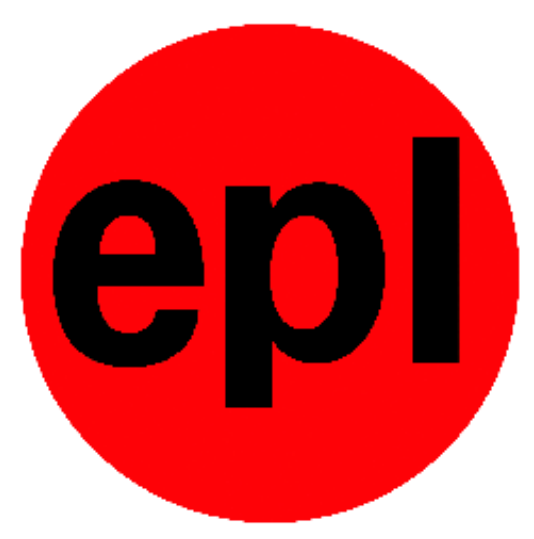

\title{
A LETTERS JOURNAL EXPLORING THE FRONTIERS OF PHYSICS
}

\section{OFFPRINT}

\section{Direct measurement of the thermomechanical Lehmann coefficient in a compensated cholesteric liquid crystal}

\author{
P. Oswald and A. Dequidt \\ EPL, 83 (2008) 16005
}

Please visit the new website

www.epljournal.org 


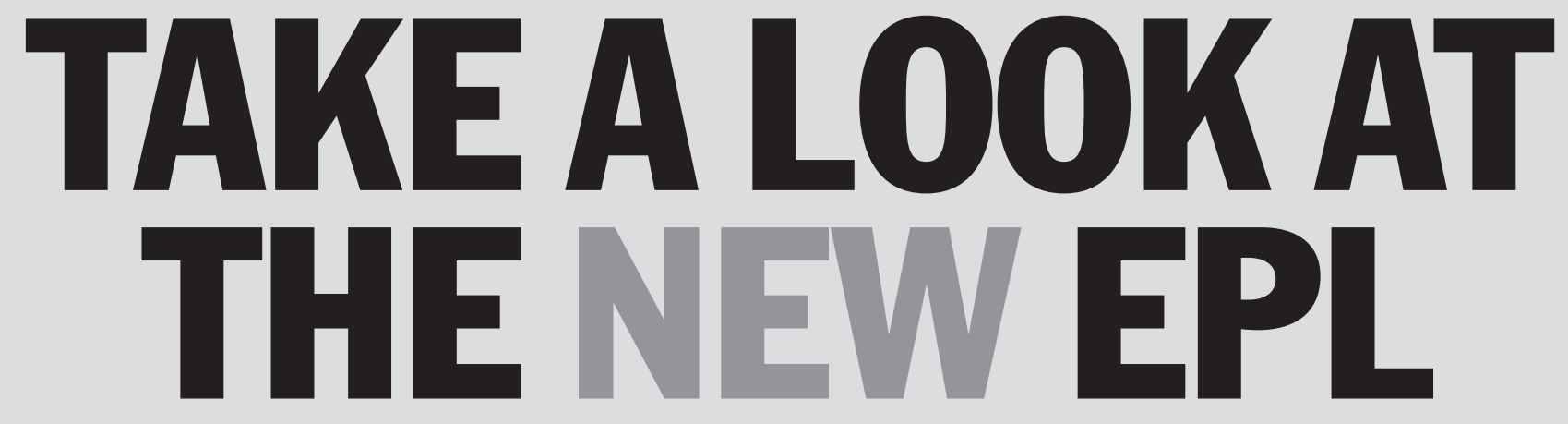

\section{Europhysics Letters (EPL) has a new online home at www.epljournal.org}

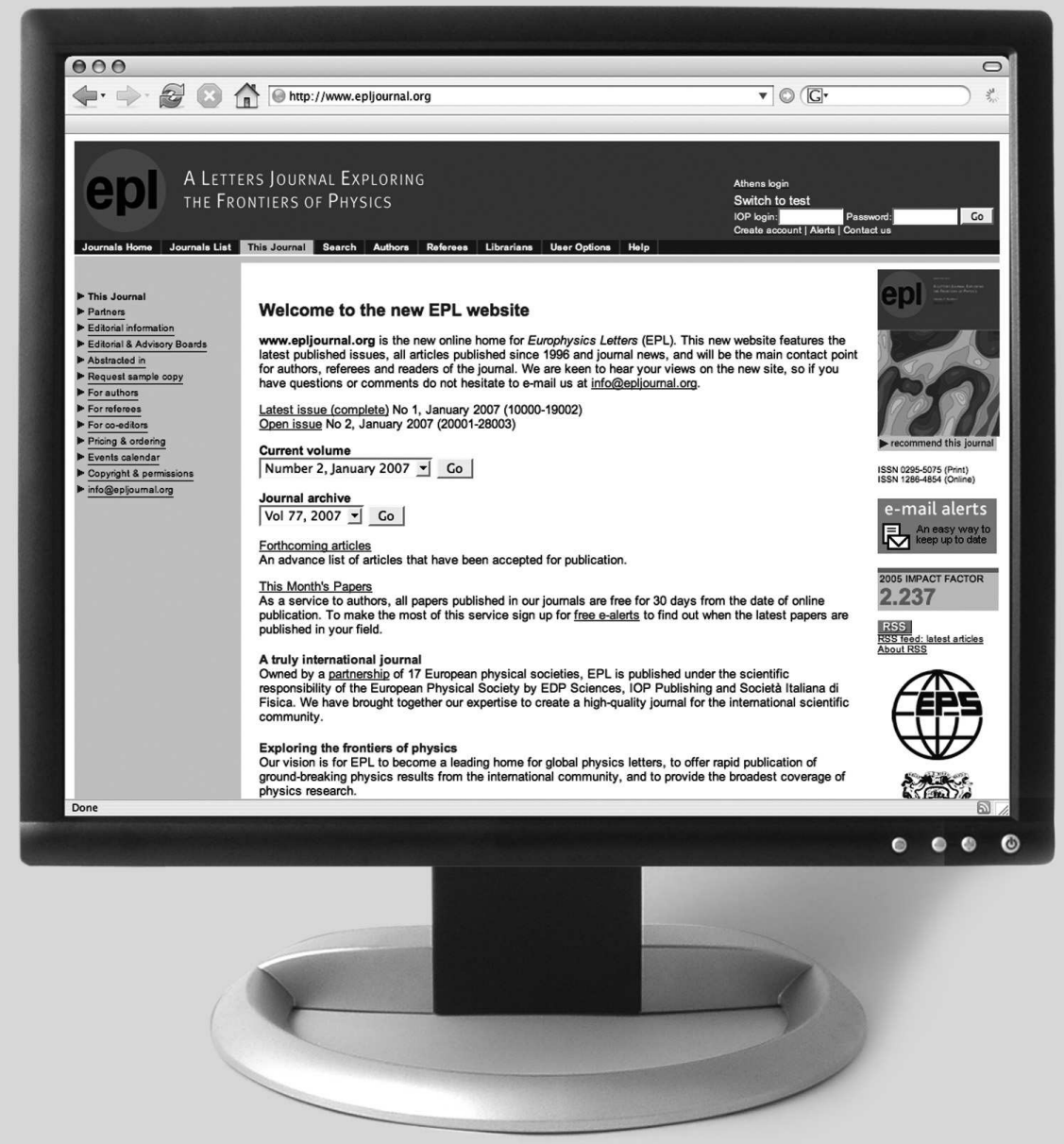

Take a look for the latest journal news and information on:

- reading the latest articles, free!

- receiving free e-mail alerts

- submitting your work to EPL 


\title{
Direct measurement of the thermomechanical Lehmann coefficient in a compensated cholesteric liquid crystal
}

\author{
P. Oswald ${ }^{(a)}$ and A. DEQUidT \\ Université de Lyon, Laboratoire de Physique, École Normale Supérieure de Lyon, CNRS - 46 Allée d'Italie, \\ 69364 Lyon, France, EU
}

received 10 March 2008; accepted in final form 21 May 2008

published online 16 June 2008

PACS 61.30.-v - Liquid crystals

PACS 05.70.Ln - Nonequilibrium and irreversible thermodynamics

PACS 65.40.De - Thermal expansion; thermomechanical effects

\begin{abstract}
The thermomechanical Lehmann coefficient $\nu$ is directly measured as a function of temperature in a compensated cholesteric liquid crystal. The method consists of observing the continuous rotation of the director in samples treated for planar sliding anchoring when a temperature gradient is applied perpendicularly to the director. The main result is that there is no relationship between the Lehmann coefficient and the equilibrium twist $q$. In particular, we confirm that $\nu$ does not vanish at the compensation temperature at which $q=0$, in agreement with previous static measurements of Éber and Jánossy (Mol. Cryst. Liq. Cryst., 72 (1982) 233) and of ourselves (Europhys. Lett., 80 (2007) 26001). In addition, the sign of the Lehmann coefficient is determined by observing between crossed polarizers the sense of rotation of the extinction branches of the disclination lines.
\end{abstract}

Copyright (c) EPLA, 2008

Introduction. - In a nematic liquid crystal, the rodlike molecules tend to align along a single direction characterized by a unit vector $\vec{n}$ called the director (with $\vec{n} \Leftrightarrow-\vec{n}$ ). If one adds chiral molecules to a nematic phase, one obtains a cholesteric phase in which the director rotates around a space direction called the helical axis. In practice, there is no obvious relationship between the molecular chirality and the value of the equilibrium twist of the phase $q$ [1]. For this reason, there exist materials in which $q$ can vanish and change sign at a given temperature called the compensation temperature $T_{c}$.

Chirality can also lead to strange phenomenon in hydrodynamics. One of the most spectacular was revealed in 1900 by Otto Lehmann who observed that the texture of particular cholesteric droplets sandwiched between two glass plates could be put into motion when heated from below $[2,3]$. This phenomenon was explained qualitatively 68 years later by Leslie [4], who showed that the absence of inversion symmetry in a cholesteric phase allows the existence of an internal torque driven by a temperature gradient, of expression $[5,6]$

$$
\vec{\Gamma}_{\text {Lehmann }}=-\nu \vec{G}_{\perp}
$$

\footnotetext{
(a) E-mail: patrick.oswald@ens-lyon.fr
}

where $\vec{G}_{\perp}=(\vec{n} \times \vec{G}) \times \vec{n}$ is the component of the temperature gradient $\vec{G}=\vec{\nabla} T$ perpendicular to $\vec{n}$ and $\nu$ the thermomechanical Lehmann coefficient.

Suppose now that the director can freely rotate at the boundaries limiting the sample. It comes immediately from the previous equation that the helix of a cholesteric liquid crystal must rotate at a constant angular velocity $\omega$ when it is subjected to a temperature gradient parallel to its axis. This velocity can be easily obtained by equilibrating the Lehmann and viscous torques and reads

$$
\omega=-\nu G / \gamma_{1},
$$

where $\gamma_{1}$ is the rotational viscosity.

Recently, we reported the first preliminary observation of the continuous Lehmann rotation of the cholesteric helix under the action of a temperature gradient $[7,8]$. This experiment was made possible thanks to the development of a new surface treatment allowing for planar and sliding anchoring of the molecules at the glass plates limiting the sample [9]. On the other hand, it was just a feasibility experiment because of the lack of an appropriate setup to impose a controlled temperature gradient.

In this article, we fill this gap and we perform the first direct and quantitative measurements of the Lehmann coefficient in a compensated cholesteric as a function of 
temperature. We emphasize here that our measurements were done on both sides of the compensation point to settle the following question: does $\nu$ vanish and change sign at $T_{c}$ as proposed by theorists $[10,11]$ or can $\nu$ be different from 0 at $T_{c}$ as found experimentally by Éber and Jánossy [12-14] and more recently by ourselves $[7,8]$ by using a static measurement method (which only applies at $\left.T_{c}\right)$ ?

Note for completeness that thermomechanical effects also exist in deformed nematics [15]. These effects are described by twelve thermomechanical coefficients $\xi_{i}$ $(i=1, \ldots, 12)$, which, when divided by the typical length over which the director field is distorted, are formally equivalent to the Lehmann coefficient $\nu$. In principle, these terms also exist in cholesterics when the helical structure is distorted, but we shall show later that their contribution is negligible in our experiment.

The plan of the article is the following. In the second section, we describe the sample preparation and our experimental setup. Then, we report our experimental results concerning the angular rotation velocity of the director as a function of the temperature and of the temperature gradient. In the next section, we show how to determine the sign of the Lehmann coefficient by observing the defects. The value of the Lehmann coefficient at the compensation temperature is then given and the importance of the nematic thermomechanical term is discussed. Finally, we draw conclusions in the last section.

Sample preparation and experimental setup. - Our liquid crystal is a mixture of 4-n-octyloxy$4^{\prime}$ cyanobiphenyl (8OCB from Synthon Chemicals $\mathrm{GmbH} \& \mathrm{Co}$ ) and of cholesteryl chloride (CC from Aldrich) in proportion $1: 1$ in weight with a compensation point at $58^{\circ} \mathrm{C}$ and a clearing point at $66^{\circ} \mathrm{C}$. The $8 \mathrm{OCB}$ was purified by A. Żywocińsky and the $\mathrm{CC}$ was used without further purification. The mixture was filtered in the cholesteric phase through $0.2 \mu \mathrm{m}$ nylon filter to remove dust particles. The sample is sandwiched between two glass plates. The bottom plate is covered with two ITO layers separated by a $2 \mathrm{~mm}$ wide central band. The two ITO layers are connected to a high-voltage power supply which allows us to impose a horizontal electric field to the liquid crystal within the gap separating the two ITO layers. The two plates are treated for planar sliding anchoring with the polymercaptan hardener of an epoxy glue (Structalit 7 from Eleco) [9]. The latter is dissolved in the 2-butanone (5\% in mass) and spread by spin-coating on the plates. It is then held at $60^{\circ} \mathrm{C}$ for one hour under vacuum in order to evaporate the solvent. Nickel wires of calibrated diameters are used as spacers to fix the sample thickness $(25 \mu \mathrm{m}$ in the present experiments). The liquid crystal is introduced by capillarity between the plates. Finally, the sample is sealed on the sides with the epoxy glue.

In order to impose a temperature gradient to the sample, a new setup was designed and constructed. It

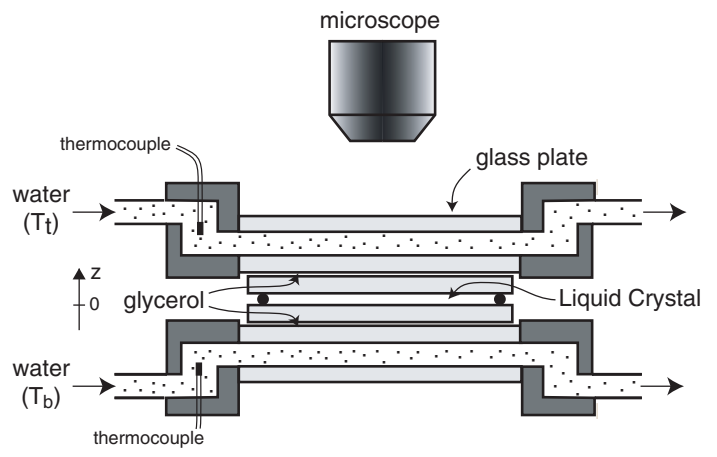

Fig. 1: Experimental setup. The $z$-axis is vertically oriented towards the top so that $G>0$ when $T_{t}>T_{b}$.

is shown in fig. 1. The sample is sandwiched between two transparent ovens regulated in temperature within $\pm 0.01^{\circ} \mathrm{C}$ owing to two separated recirculating water baths. Two thin layers of glycerol are introduced between the sample and the ovens in order to enhance thermal contacts. With a home-made temperature sensor [9], we checked that the sample temperature was given by $\left(T_{b}+T_{t}\right) / 2$ within $\pm 0.1{ }^{\circ} \mathrm{C}$ whatever the temperatures chosen for the top and bottom ovens $\left(T_{t}\right.$ and $T_{b}$, respectively). The two ovens are mounted on a precision $X Y$ stage of a polarizing Leica microscope allowing us to observe the complete sample. A video camera allows us to capture up to 30 images/s with a resolution of $1380 \times 1024$ pixels. In the following, we shall denote by $\Delta T$ the difference $T_{t}-T_{b}$. This quantity is directly proportional to the imposed temperature gradient $G$ which will be evaluated later.

Experimental results. - The first step was to determine precisely the compensation temperature $T_{c}$. As the latter is known within $\pm 0.5{ }^{\circ} \mathrm{C}$, we started by fixing the temperature of the ovens at the presumed temperature. We then applied a large voltage (typically, 500 Vrms at frequency $f=10 \mathrm{kHz}$ ) between the two electrodes. Because the liquid crystal has a positive dielectric anisotropy $\left(\varepsilon_{a} \approx 5[16]\right)$, the director orients parallel to the electric field in the region lying between the two electrodes. As a consequence, the sample appears black in this region between crossed polarizer and analyzer when the polarizer is either parallel or perpendicular to the electric field. We then switch off the voltage. If the temperature is different from $T_{c}$ the director field winds again and the extinction disappears. On the other hand, the extinction persists if the temperature is equal to $T_{c}$ as the sample is "nematic" at this temperature. Thus, one can determine $T_{c}$ by looking for the temperature at which the extinction persists. In our experiments, we found $T_{c}=57.8 \pm 0.1^{\circ} \mathrm{C}$.

Once $T_{c}$ was determined, we started by increasing the temperature difference between the two ovens while maintaining the sample temperature equal to $T_{c}$. Doing this we observed that the transmitted intensity between 


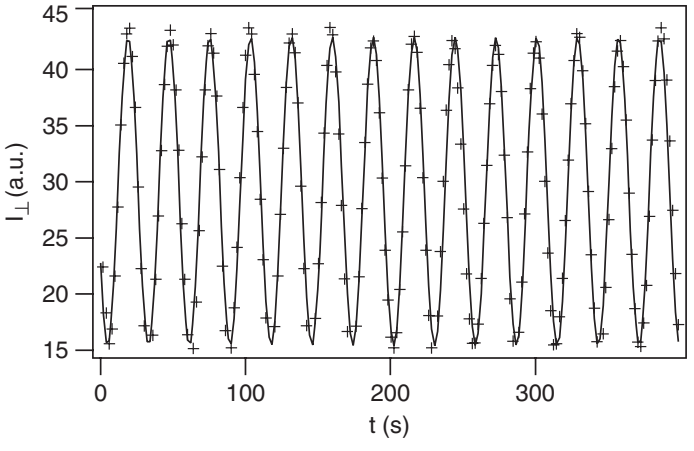

Fig. 2: Intensity measured between crossed polarizers as a function of time after the electric field has been removed. The solid line is the fit to a sine function. $T=T_{c}$ and $\Delta T=40^{\circ} \mathrm{C}$.

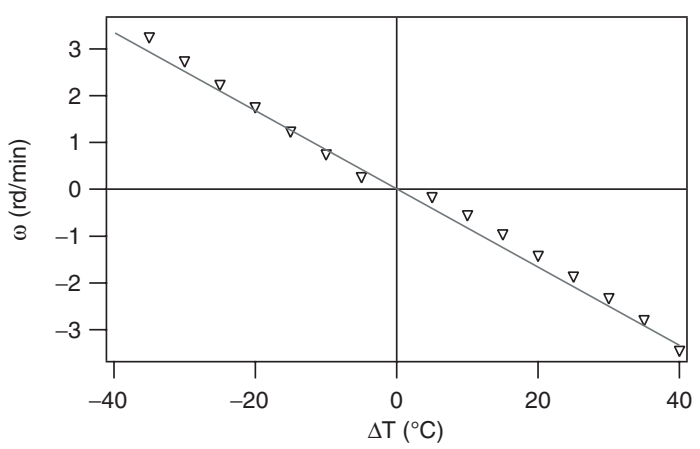

Fig. 3: Angular rotation velocity as a function of the temperature difference. $T=T_{c}$.

crossed polarizer and analyzer began to oscillate in time immediately after the field was removed. This oscillation is clearly due to the Lehmann rotation of the director. Note that the intensity oscillates at the angular velocity $4 \omega$ because it passes through a minimum each time the director is parallel or perpendicular to the polarizer. Figure 2 shows the intensity as a function of time. As expected, it is very well fitted with a sine function, which is the sign that the director regularly rotates on the two glass plates.

In fig. 3 we reported the director angular velocity $\omega$ as a function of the temperature difference $\Delta T$. This graph shows that the angular velocity (the sign of which can be determined by observing the sense of rotation of the extinction branches of the defects as we shall show in the next section) is proportional to $\Delta T$ (and, thus, to the temperature gradient $G$ ) in agreement with eq. (2).

Finally, we measured $\omega$ as a function of the sample temperature $T=\left(T_{b}+T_{t}\right) / 2$ while maintaining $\Delta T$ constant. Our results are reported in fig. 4. They show unambiguously that the angular velocity and, thus, the Lehmann coefficient $\nu$, do not vanish at $T_{c}$, contrary to the equilibrium twist $q$.

Sign of the Lehmann coefficient. - The intensity curves as a function of time like that shown in fig. 2 only give the absolute value of $\omega$. A method to determine the sign of $\omega$ consists of observing between crossed polarizers

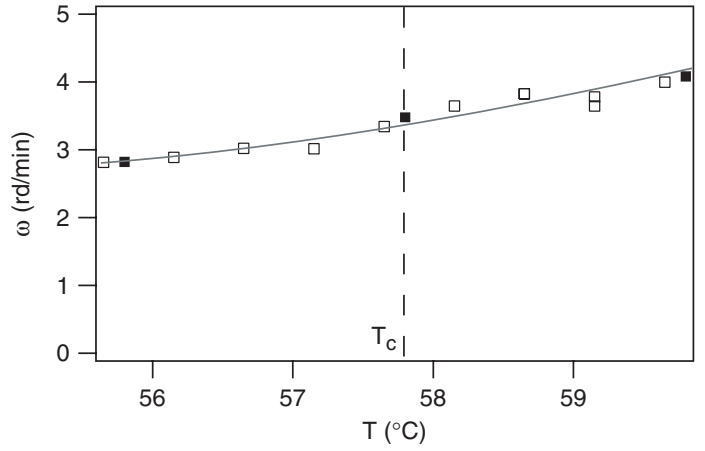

Fig. 4: Angular velocity as a function of the sample temperature for a fixed temperature difference $\Delta T=-40^{\circ} \mathrm{C}$. The open and filled squares correspond to two different samples of thickness $d=25 \mu \mathrm{m}$.

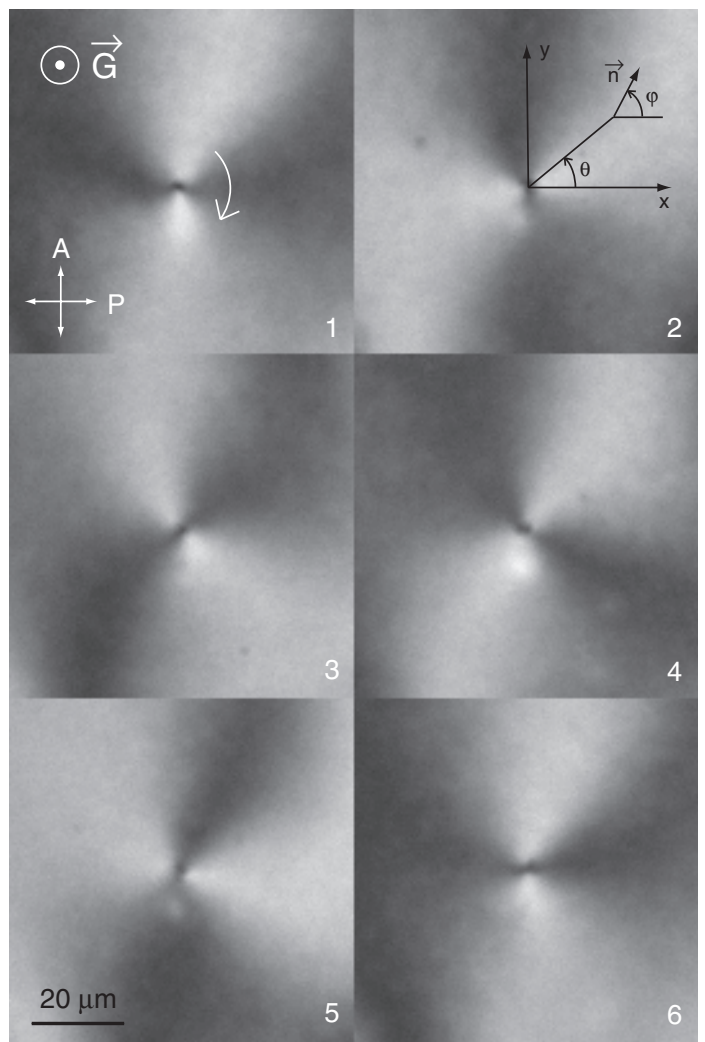

Fig. 5: The two extinction branches of a $-1 / 2$ disclination line rotate clockwise when the temperature gradient is positive. The interval time between two photographs is $11 \mathrm{~s} . T=T_{c}$ and $\Delta T=38.5^{\circ} \mathrm{C}$.

the sense of rotation of the extinction branches of the wedge disclination lines. The latter form in large number when the sample is cooled down from the isotropic liquid. In practice, most of the lines are of topological strength $m= \pm 1 / 2$. Such a defect is shown in fig. 5. In this example, $m=-1 / 2$ because the branches turn in the opposite direction of the polarizers. In order to determine at which angular velocity the branches must rotate under the action of the Lehmann effect, let us give the expression of the angle $\varphi$ between the director and the $x$-axis around the 
core of a disclination line. Because the director rotates with the angular velocity $\omega$, one has for a disclination line of strength $m$ situated at the origin

$$
\varphi=m \theta+\omega t
$$

where $\theta$ is the polar angle (fig. 5). If the polarizer (respectively, the analyzer) is parallel to the $x$-axis (respectively, the $y$-axis), there is extinction at places where $\varphi=0$ and $\pi / 2$. This defines the equation of the two extinction branches

$$
\theta=-\frac{\omega}{m} t \quad \text { and } \quad \theta=\frac{\pi}{2 m}-\frac{\omega}{m} t .
$$

These equations show that the branches of a $-1 / 2$ (respectively, $+1 / 2$ ) disclination line must rotate with the angular velocity $2 \omega$ (respectively, $-2 \omega$ ).

Let us now return to fig. 5 showing a $-1 / 2$ defect. In this example, $T=T_{c}, G>0$, and the two extinction branches rotate clockwise which means that $\omega<0$. From this observation and according to eq. (2) we deduce that $\nu>0$ at $T_{c}$. We checked that the same result holds in the whole investigated range of temperature (about $2{ }^{\circ} \mathrm{C}$ on both sides of $T_{c}$ ).

Value of the Lehmann coefficient at the compensation temperature. - In practice, eq. (2) must be corrected because the director does not slide freely on the polymercaptan layers, but experiences a viscous surface torque of expression $\Gamma_{S}=-\gamma_{S} \vec{n} \times \frac{\partial \vec{n}}{\partial t}$ [9]. In this condition, $\gamma_{1}$ must be replaced in this equation with a larger effective viscosity $\gamma_{1}^{*}$ which takes into account the dissipation at the surfaces. To show this result and explicitly calculate $\gamma_{1}^{*}$, let us write the basic equations of the problem.

Let $\varphi$ be the angle between the director and the $x$-axis parallel to the plates. In the bulk, the torque equation reads

$$
K_{2} \frac{\partial^{2} \varphi}{\partial z^{2}}-K_{2} \frac{\partial q}{\partial z}=\nu G+\gamma_{1} \frac{\partial \varphi}{\partial t}
$$

where $K_{2}$ is the twist constant and $q$ the equilibrium twist. Note that in this equation, we took into account the temperature variation of $q$ along $z$ but neglected the $K_{2}$ variation. This is justified around $T_{c}$ because only $q$ is strongly dependent on the temperature.

At the bottom plate, the surface torque equation reads

$$
-K_{2}\left(\frac{\partial \varphi}{\partial z}-q(0)\right)+\gamma_{S} \frac{\partial \varphi}{\partial t}=0 \text { at } z=0
$$

while at the top plate it reads

$$
+K_{2}\left(\frac{\partial \varphi}{\partial z}-q(d)\right)+\gamma_{S} \frac{\partial \varphi}{\partial t}=0 \text { at } z=d
$$

To solve these equations, we need to know the $q(T)$ variation. In previous works [7,8], we showed that $q$ changes linearly with temperature around $T_{c}$ within an excellent approximation. This allowed us to write that $\frac{\partial q}{\partial z}=\frac{\mathrm{d} q}{\mathrm{~d} T} G$ in eq. (5) and that $q(d)=q(0)+\frac{\mathrm{d} q}{\mathrm{~d} T} G d$ in eq. (7) where $\frac{\mathrm{d} q}{\mathrm{~d} T}$ is a constant. In this condition, the solution can be written in the form $a z+b z^{2}+\omega t$, where $a$ and $b$ are two constants describing the spatial distortions of the helix, while $\omega$ is its angular velocity. A straightforward calculation yields

$$
\omega=-\frac{\nu}{\gamma_{1}^{*}} G
$$

where $\gamma_{1}^{*}+\gamma_{1}+2 \gamma_{S} / d=0$ is an effective viscosity larger than the bulk viscosity $\gamma_{1}$. This equation generalizes eq. (2) as it includes the surface viscous dissipation.

It turns out that $\gamma_{1}$ and $\gamma_{S}$ were measured in a previous work at the compensation temperature: $\gamma_{1}=0.075 \mathrm{Pas}$ and $\gamma_{S}=3.2 \times 10^{-7} \mathrm{Pasm}[9]$. As a consequence, the only quantity which remains to determine to estimate the Lehmann coefficient is the temperature gradient $G$ across the liquid crystal layer. In practice, the glass plates are much thicker than the glycerol and liquid crystal layers. In these conditions, we can neglect in a first approximation the temperature variations across them, which gives

$$
G \approx \frac{\Delta T}{4 e} \frac{\kappa_{g}}{\kappa_{L C}}
$$

where $e$ is the thickness of a glass plate $(1.1 \mathrm{~mm})$ and $\kappa_{g}$ and $\kappa_{L C}$ the thermal conductivities of the glass and of the liquid crystal, respectively.

To measure the ratio $\kappa_{g} / \kappa_{L C}$ we replaced the upper glycerol layer with a thin layer of pure 80CB. Our material has a nematic-to-smectic A phase transition at temperature $T_{N A}=66.2^{\circ} \mathrm{C}$. As this transition is easy to detect under the microscope, it can therefore be used to fix a temperature. To measure the conductivity ratio, we thus changed the temperatures $T_{b}$ and $T_{t}$ while maintaining the temperature of the $8 \mathrm{OCB}$ layer exactly at $T_{N A}$. In this condition, it can be shown that $T_{b}$ and $T_{t}$ must satisfy the following equation:

$$
T_{b}=\frac{4 x+1}{x} T_{N A}-\frac{3 x+1}{x} T_{t},
$$

where $x=\frac{e}{d} \frac{\kappa_{L C}}{\kappa_{g}}$. Thus, measuring $T_{b}$ as a function of $T_{t}$ allows us, in principle, to measure the ratio $\kappa_{g} / \kappa_{L C}$. To increase the sensibility of the measurement, $x$ must be as small as possible. For this reason, we used a thick sample of the 8OCB-CC mixture: $d=550 \mu \mathrm{m}$. Our experimental results are shown in fig. 6 . The best fit to eq. (10) gives $x=0.29$ from which we calculate $\kappa_{g} / \kappa_{L C} \approx 7$.

We can now calculate the value of $\nu$ at $T_{c}$ from eqs. (8), (9) and knowing that $\omega \approx 3.4 \mathrm{rad} / \mathrm{min}$ when $\Delta T=$ $40{ }^{\circ} \mathrm{C}$ and by using the previous values of $\gamma_{1}, \gamma_{S}$ and of the ratio $\kappa_{g} / \kappa_{L C}$. This gives

$$
\nu \approx 1 \times 10^{-7} \mathrm{~kg} \mathrm{~K}^{-1} \mathrm{~s}^{-2} .
$$

This value is twice as small as the value we found before by a static method $[7,8]$. This difference can come from the fact that we overestimate the temperature gradient by neglecting the thermal losses as well as 


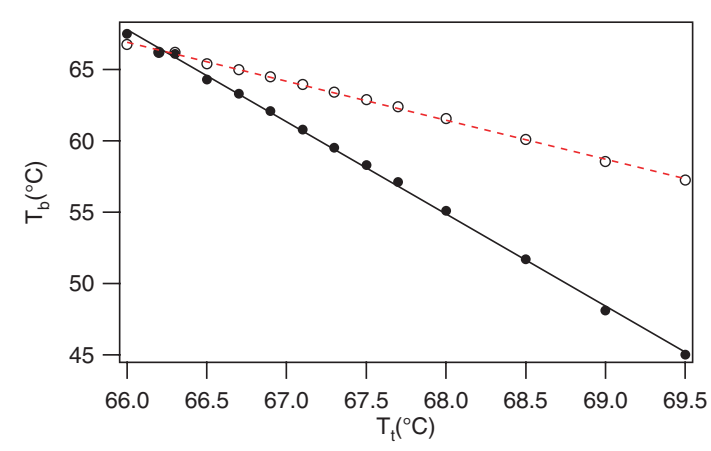

Fig. 6: Bottom temperature as a function of the upper temperature and the best fit to eq. (10) (solid line). The open circles give the temperature in the middle of the liquid crystal layer.

the influence of the two glycerol layers. In addition, the ratio of the two conductivities has been measured at a larger temperature than $T_{c}$ in a disoriented sample which generates a supplementary error. Finally, it is possible that we underestimate $\gamma_{S}$ and, thus, the effective viscosity $\gamma_{1}^{*}$, because we did not use the same glue sample as in ref. [9]. For these reasons, the value of $\nu$ given before must be regarded as giving only a good order of magnitude.

Before concluding, we return to the nematic thermomechanical terms [15] which we mentioned in the introduction and neglected so far. This is not strictly correct because the helix deforms when it rotates under the action of the temperature gradient. This effect introduces in the r.h.s. of eq. (5) an additional term of the type $\xi\left(\frac{\partial \varphi}{\partial z}-q\right) G$. Taking into account this term is possible but leads to a non-linear relationship between the angular velocity $\omega$ and the temperature gradient $G$. This is not observed within experimental errors (see fig. 3), which suggests that this term is indeed negligible in our experiments. More precisely, it can be shown from eq. (5) that this term can be neglected if $\xi<\frac{K_{2}}{d G}$. This inequality shows that the smaller the temperature gradient or the thickness, the weaker the effect of the thermomechanical nematic term. In our experiment, $d=25 \mu \mathrm{m}$ and the maximum temperature gradient used was $G \approx 6 \times 10^{4}{ }^{\circ} \mathrm{C} / \mathrm{m}$. Knowing that $K_{2} \approx 3 \times 10^{-12} \mathrm{~N}[7,8]$ at $T_{c}$, we estimate that $\xi<3 \times 10^{-12} \mathrm{~kg} \mathrm{~m} \mathrm{~K}^{-1} \mathrm{~s}^{-2}$ in our CC-8OCB mixture. This conclusion is not incompatible with a previous estimate of a similar coefficient in the liquid crystal 5CB (pentylcyanobiphenyl) in which $\xi \sim 10^{-12} \mathrm{~kg} \mathrm{~m} \mathrm{~K}^{-1} \mathrm{~s}^{-2}$ [17].

Conclusions. - To summarize, our experiment shows directly for the first time the continuous Lehmann rotation of a cholesteric helix when it is subjected to a temperature gradient. Owing to a new setup, we were able to measure its angular velocity and to show that it is proportional to the temperature gradient in agreement with the theory. We also determined the absolute value of the Lehmann coefficient $\nu$ and its sign by observing the sense of rotation of the extinction branches of the disclination lines. More importantly, we found that the Lehmann coefficient remains positive on both sides of the compensation point and so does not vanish nor changes sign at this point, contrary to the equilibrium twist $q$. This result agrees with static measurements of $\nu$ at $T_{c}[7,8,12-14]$ and contradicts the usual idea according to which $\nu$ must be proportional to $q[10,11]$. The reason for this counterintuitive result is that the cholesteric phase has never inversion symmetry, even at $T_{c}$ when it is unwound and has a nematic-like structure, because of the chirality of the molecules. It is for similar symmetry reason that a chiral isotropic liquid (as, for instance, a solution of saccharose in water) has rotatory power. As a consequence, there is no obvious relationship between the Lehmann coefficient and the equilibrium twist. From this point of view it would be interesting to investigate how these two quantities change with the concentration and the nature of the chiral molecules. Such experiments are now in progress. Finally, note that nematic thermomechanical effects are too small to be detected in this experiment.

$$
* * *
$$

We thank F. VitToz for the construction of the experimental setup.

\section{REFERENCES}

[1] Harris A. B., Kamien R. D. and Lubensky T. C., Rev. Mod. Phys., 71 (1999) 1745.

[2] Lehmann O., Ann. Phys. (Leipzig), 2 (1900) 649.

[3] Lehmann O., Flüssige Kristalle und ihr Scheinbares Leben (Verlag von Leopold Voss, Leipzig) 1921.

[4] Leslie F. M., Proc. R. Soc. London, Ser. A, 307 (1968) 359.

[5] De Gennes P.-G. and Prost J., The Physics of Liquid Crystals (Oxford University Press, Oxford) 1995.

[6] Oswald P. and Pieranski P., Nematic and Cholesteric Liquid Crystals: Concepts and Physical Properties Illustrated by Experiments (Taylor \& Francis, CRC press, Boca Raton) 2005.

[7] Dequidt A. and Oswald P., Europhys. Lett., 80 (2007) 26001.

[8] Dequidt A., Żywociński A. and Oswald P., Eur. Phys. J. E, 25 (2008) 277.

[9] Oswald P., Dequidt A. and Żywociński A., Phys. Rev. E, 77 (2008) 061703.

[10] Pleiner H. and Brand H. R., Mol. Cryst. Liq. Cryst. Lett., 5 (1987) 61.

[11] Pleiner H. and Brand H. R., Mol. Cryst. Liq. Cryst. Lett., 5 (1988) 183.

[12] Éber N. and Jánossy I., Mol. Cryst. Liq. Cryst. Lett., 72 (1982) 233.

[13] Éber N. and Jánossy I., Mol. Cryst. Liq. Cryst. Lett., 102 (1984) 311.

[14] Éber N. and Jánossy I., Mol. Cryst. Liq. Cryst. Lett., 5 (1988) 81.

[15] Akopyan R. S. and Zel'dovich B. Ya., Sov. Phys. JETP, 60 (1984) 953.

[16] Dequidt A. and Oswald P., Eur. Phys. J. E, 24 (2007) 157.

[17] Akopyan R. S., Alaverdian R. B., Santrosian E. A. and Chilingarian Y. S., J. Appl. Phys., 90 (2001) 3371. 